\title{
Reassessment of the Concept of Momentum in Econophysics
}

\author{
Mario J. Pinheiro and Mario R. A. Pinheiro
}

\begin{abstract}
We examine the most basic feature of the economic process - momentum - under the point of view of analogies with physical laws, as they were reformulated recently [1]. Our approach is applied with minimal assumptions and we conclude that the inclusion of entropy as an effective variable in econophysics may bring a new vision of economic progress and the possibility to harness economic waves as a means to transport development from rich to poor countries using trade and technological progress. A new technical indicator for the stock market is proposed offering double opportunities on enter and exit trades, when compared to the Relative Strength Index usually used in analysis of financial markets.
\end{abstract}

Keywords - Black-Scholes formula, Econophysics; Options; Quantitative finance; Risk measures; Standard deviation; Technological progress.

\section{INTRODUCTION}

A new formulation of classical mechanics which differs in its essential philosophy from the Newtonian Mechanics, as commonly taught in engineering and science [1]-[3] is applied to economics and finance. Based on the novel concepts introduced, we suggest a new definition of financial momentum. We expect that our proposal might enhance the role of econophysics in social, economic, and industrial progress. In consequence of the financial crisis of 2007-8, we identify two kinds of economic mechanics: i) the unconventional monetary policy based on quantitative easing, i.e., the injection of new money into the money supply by a central bank; i) conventional economics, based on finite energy sources and technological progress. We call the unconventional economic system as digital economy and as it is not based on the production of new material commodities but mostly on AI. Our interest here is centered on conventional economics.

Then the main topic of our manuscript and the encountered problems are analyzed in the light of the novel concepts introduced and discussed in [1]-[3] where a variational model embedded in the description of a physical system the quantity energy and entropy. To discuss, and introduce our proposals, the manuscript is organized as follows: Section II introduces the basic aspects of the physical model translated to the economic space; Section III suggests a new meaning for the option volatility trends as the inverse of the transaction (strike price/asset price) in the Black \& Scholles equation; Section IV proposes a modified form of the Schrödinger equation on the space of prices of shares and a new technical indicator for momentum; Section V illustrates how the concept of economic momentum can enlighten the dynamic among a set of countries in economic exchanges; Section VI proposed a mechanism of induced waves between countries enhancing economic progress. In the end, we conclude the paper with the main findings.

\section{BRIEF DESCRIPTION OF THE PHYSICAL MODEL}

To lay down the rationale of the foregoing considerations on econophysics, we begin by introducing the basic physical model. The mathematical representation of physical systems are defined by the following system of equations [1]:

- Canonical momentum :

$$
T \frac{\partial \bar{S}}{\partial \mathrm{p}} \geq-\frac{\mathrm{p}}{m}+\frac{q}{m} \mathrm{~A}+v_{e}+[\omega \times r]
$$

Submitted on November 01, 2021

Published on November 04, 2021.

Mario J. Pinheiro, Departamento de Física, Universidade de Lisboa, Portugal.

(e-mail: mpinheiro@tecnico.ulisboa.pt)

Mario R. A. Pinheir, EconoDynamics Group, Portugal.

(e-mail: mrapinheiro98@gmail.com) 
- Fundamental equation of motion:

$$
T \frac{\partial \bar{S}}{\partial \mathrm{r}} \geq \nabla U+m \frac{\partial \mathrm{v}}{\partial t}
$$

Eq. (1) returns the quantity of motion, a measure of the vector velocity (with geometric nature), and the quantity of matter (with energetic content). However, Eq. (1) describes a radically different brand of a balance of momentum when compared to the Newtonian concept of force and momentum, because it predicts mutual interaction between interacting systems, self-regulating exchange of energy (see also, [1]). The Newtonian conception of work as the dominating force of a physical system or the society, requires the other side of the coin, the presence and actuation of entropy as the representation of "interactions" with the medium, not necessarily constraining a system to be submitted to external forces.

Eq. (2) supplies the fundamental equation of dynamics and has the form of a general local balance equation holding as source term the spatial gradient of entropy, $\nabla_{a} S>0$. At thermodynamic equilibrium, the total entropy of the body, or physical system, has a maximum value and the equality holds, a characteristic in general absent in the conventional dynamical determinism of Newtonian formulation (although Newton admits friction as a source of asymmetry). The men- tioned self-regulating mechanism of angular and linear momentum is represented by the last term of Eq. (1) and stretches the validity of Newton's fundamental equation of motion to accelerated (non-inertial) frames of reference (see [1]). In Eqs. (1)-(2) it emerges one of the most relevant thermodynamic quantity, the temperature $T$. However, in this work, we don't discuss the remarkable diversified phenomena of the conversion of heat into work (and vice-versa) and what it would be the analogs in the econophysical context [4].

The set of Eqs. (1)-(2) open a significant change in the so-far classical paradigm of dynamics, and as well electrodynamics (see [1], [2]), since new physics may be brought by the set of two first-order differential equations, related to the transaction between the tendency of energy to attain a minimum, whilst entropy attempts to maximize its value. According to the proposed variational method, Eq. (2) leads to the fundamental equation of (classical) dynamics, now presented under the form (see, [3] for examples and applications):

$$
m \frac{\partial \mathrm{v}}{\partial t}=\mathrm{F}^{e x t}-\nabla\left(\frac{J^{2}}{2 I}-\omega \cdot J+T \Delta S\right)
$$

It is a consequence of requiring that the energy and entropy attain an optimal balance. This new principle is, therefore, different from Hamilton's principle since this last one is equivalent to $\mathrm{F}=m a$ and it does not contain the action of dissipative forces nor the free energy term, source of nonlinearities, and retroaction.

The external force term can be a deterministic or fluctuating force, but cannot be entropy-dependent. A central role is played by the gradient of rotational energy (and free energy) which drives the system from one state to another. The system is not enslaved by the external force $\mathrm{F}^{\text {ext }}$, which we may envisage as an input to the system, but can respond to changes by converting linear or angular motion into angular motion, which we may consider the system output, affecting dynamically the system, as illustrated in Fig. 1 of [2]. Moreover, according to Eq. (3), the motion will not necessarily follow in a straight line and in the direction of the external force (see Section II, A). Angular momentum acts as a damper to dissipate a disturbance, a well-known redressing mechanism in biomechanics and robotics. The free energy term is essential to study the thermomechanical behavior of any continuous media.

In the context of the above framework, it appears to us that in the economic sphere, three types of driving forces (in the sense of agents of economic behavior) may actuate on a system:

1. An external driving force (e.g.: regulations, supplier and sourcing instability, competitor moves, technology, cus- tomer preferences);

2. A "force" that is connected to triangular arbitrage (a trading strategy that exploits the arbitrage opportunities that exist among three currencies in a foreign currency exchange);

3. A "force" that is pushed by the Economic Complexity Index (or level of technology) i.e., an integrated measure of the productive aptitudes of extensive economic systems, normally cities, regions, or countries.

\section{A. The econophysical Model}

$T$, playing here the role of the kinetic temperature in physical systems, is the analog of the "level of technology", and $W=T \Delta S$ is the analog of the "product of technology". Money is the "agent of transformation" of the economic engine, a class of fluid (similarly, in a locomotive, we have water, subordinate to heating and two different temperatures). Therefore, money is not the holy grail but merely 
the agent of transformation.

Nicolas Copernicus was not simply an astronomer who realized the earth revolved around the sun; he also appears up with the original Quantity Theory of Money (QTM) which is centered around the concept of velocity of money. "An excessive quantity of money," according to Copernicus, "should be avoided." Consequently, a low velocity of money would cause deflation, and a high velocity would cause inflation, but not always. According to Copernicus, and nowadays also known as the Fisher's equation of exchange, economic momentum is defined by the equation:

$$
M V_{T}=P_{T} T
$$

or $M V_{T}=P_{T} Y$, where $M$ expresses the total amount of money in circulation on an economic system (for a given period, say, a year), $V_{T}$ is the transactions velocity of money (the number of times money changes hands in a given time), $P_{T}$ is the general price level associated with transactions for the economy for the assigned period (say, a year), $T$ is transactions or an index of the real value of aggregate transactions, and $Y$ is the real GDP (the real product of the economy).

Pursuing into the analogy with Eq. (3) we would expect that the "canonical momentum" in the context of finances would be described by the equation:

$$
P=M V_{T}-T \frac{\Delta S}{\Delta V_{T}}
$$

It recommends that the "monetary momentum" is proportional to the money supply by the velocity of money minus the product of the technological level by the rated velocity of the product of technology. Monetary Momentum is not the ("mechanical") product of $M V_{T}$ but it depends, further, on how the product of technology shifts with the velocity of money; technological innovation has an impact on the velocity of money. It also signifies that the product of technology (understood as material, tangible objects that have been designed by people and developed within a technological practice to serve particular functions) must decrease with increasing spending of money.

\section{TRAding the MARKET WITH OPTIONS AND StOCKS: A NEW INTERPRETATION OF THE BlaCK- SCHOLLES EQUATION}

We propose now a balance equation for the quantity (PT) based on Eq. (3):

$$
\frac{d(P T)}{d t}=F^{e x t}-\frac{d}{d v}\left[\frac{V(P T)}{(P V)}-W\right]
$$

We may write, after simple manipulation, and including a term representing external economic driving forces $d q=\frac{F^{e x t} d t}{P T}$, Eq. (6) under form:

$$
\frac{d P}{P}=\frac{1}{P T} \frac{D}{d V}(W-T) d t-\frac{1}{T} d T+d q
$$

which is mathematically similar (but not identical, due to the re-definition of the coefficients) to the Black and Scholles equation:

$$
\frac{d P}{P}=\mu\left(S_{t}, t\right) d t+\sigma\left(S_{t}, t\right) d W_{t}+d q
$$

after assuming the following identification:

$$
\begin{gathered}
\mu\left(S_{t}, t\right)=\frac{1}{P T} \frac{d}{d V} \pi(W-T) \\
\sigma\left(S_{t}, t\right)=\frac{1}{T}
\end{gathered}
$$


Eq. ( $9 \mathrm{~b}$ ) shows that the option volatility trends as the inverse of the transaction (strike price/asset price), some evidence observed here [5]. Eq. (8) is similar to Merton model (in a class of jump-diffusion models) [6].

In Eqs. (8), (9), we seek now a more accurate meaning of them. For $\sigma$ we notice that, in this new framework, it appears the contribution of the economic complexity index [7] (via $T \Delta S$ ), the effects of triangular arbitrage opportunities (a form of interaction that takes advantage of three changes rates between three currencies, for example, US dollar to Japanese yen to Euros and back to US dollars). The triangular arbitrage is the analogue of the angular momentum in physics. In addition, considering the hyperfine-

structure term already showing up in the initial physical equation (the term $\omega \dot{\Omega}$ ), we may anticipate an interaction amid, at least, two different triangular arbitrages, for example, $\omega \rightarrow U S D-J P N-E U R-U S D$ and $\ldots \Omega \rightarrow$ Banks. Triangular arbitrage extends to cryptocurrencies in multiple exchanges in a blockchain system.

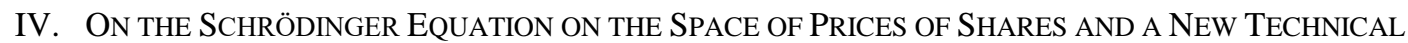 INDICATOR FOR MOMENTUM}

Eq. (3) represents a novel, and more consistent, description of a physical system [1]-[3]. Its convoluted energy with entropy and the related Schrödinger equation is given by:

$$
H \Psi=i \hbar \frac{\partial}{\partial t} \Psi=-\frac{\hbar^{2}}{2 m} \nabla^{2} \Psi+(V+J) \Psi
$$

where $H$ denotes the Hamiltonian of the system and $\Psi(S, t)$ is the wavefunction of the stock price $S$ at a given instant of time $t$. Note that we have now included not only the usual external potential $V$ but also a new term which must be the econophysics analog of the thermo-mechanical term $\left.\left[\frac{J^{2}}{2 I}-(\omega) \cdot J-\Delta F\right)\right]$. The analog of this new term is the triangular arbitrage used in the stock market. Triangular arbitrage may be used when a discrepancy occurs between three foreign currencies. As these opportunities are rare, traders only can profit from them by using advanced computer equipment and/or programs to automate the process. A trader employing triangular arbitrage would exchange an amount at one rate (EUR/USD), convert it again (EUR/GBP) and then convert it finally back to the original (USD/GBP), and assuming low transaction costs, gaining a net a profit. As noted before, the free energy term $\Delta F$ is the analog of the index of the product of technology given, for example, by the Dow Jones U.S. Technology Index (Ticker: DJUSTC), a price-weighted measurement stock market index of 30 most influential companies listed on stock exchanges in the United States.

At another level, triangular (rather than bi-lateral) cooperation boost benefits for the pivotal countries and in develop- ment beneficiaries since "While social, economic and political contexts vary from country to country, pivotal and bene- ficiary countries may share economic and geographic characteristics, cultural, historical and regional ties, and common languages" [8].

Additionally, markets have lowers and ups. This fact presses the introduction of a new indicator, the "sector rotation", a presently widely engaged theory of stock market activity. A sector rotation investment strategy involves "rotating" or shifting from sector to sector as the economy runs into the different phases of the business cycle (see, e.g., [9]). Based on these analogies and Eq. (1), the best analog of the $m[\omega \times r]$, we may propose a new technical indicator to determine the strength or weakness of a stock's price named here $\operatorname{PPS}_{\omega}^{i j}$ giving the forex or stock trend between two distinct instants of time $i \rightarrow j$ :

$$
\operatorname{PPS}_{\omega}^{i j}=100 \frac{R S}{1+R S}+\frac{1}{T}\left(P_{j}-P_{i}\right) \Delta t_{i j}
$$

Here, RS is the Relative Strength $(R S=A v g U / A v g D)$, with $A v g U$ the average of all up moves in the last $\mathrm{N}$ price bars, and $A v g D$ the average of all down moves in the last $\mathrm{N}$ price bars, $N$ denoting the period of the RSI technical indicator (see, e.g., [10]). The second term on the RHS of Eq. (11) represents the $m[\omega \times r]$ of mechanics. Fig. 1 shows the performance of the RSI ${ }_{\omega}^{i j}$ USD/JPY prices with the MACD (a), RSI(b) and $\mathrm{RSI}_{\omega}^{i j}$ (c). The introduction of sector rotation brings a more exact instant of time to enter in a buy/sell decision for an asset than other technical indicators, and, additionally, it doubles the number of 
opportunities to trade an asset (see Fig. 1 for illustration, and Appendix A with custom algorithm coded in Java). The excursion of the price inside the boundaries at 30 and 70 represent typically a sideways drift that takes place when the price of a security trades within a stable range over some period of time, with neither the bulls nor bears taking control of prices. It might be useful in a forex or stock trading strategy.

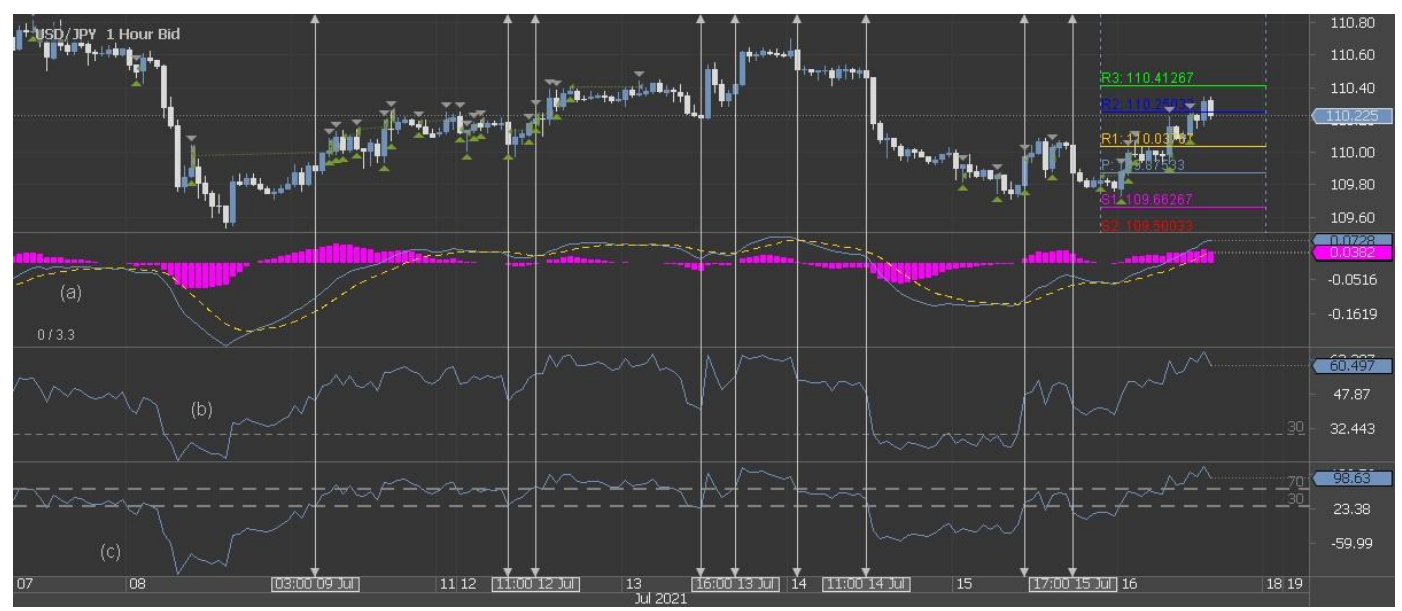

Fig. 1. Comparison between technical indicators for the USDJPY prices: MACD (a), RSI(b) and the new indicator (c).

\section{ON INTERACTING COUNTRIES}

We may envisage in econophysics an interactive "atomistic" approach. If we consider a system of, let us say, three countries in interaction, we may expect conservation of momentum (when assuming that the three form a closed system):

$$
p_{1}+p_{2}+p_{3}=\text { constant }
$$

The economical rebound of three European countries can be observed in the graphical representation of Fig. 2, and it can be seen as a real illustration of this concept.

Fig. 2 shows the evolution of the industrial production from 1990 to 2015 . At each year the sum of the industrial pro- duction of these industrialized countries is approximately constant and varies within $99 \%$. When one country augments its industrial production within the European Union, the other decreases according to Eq. (12). As the (economic) momen- tum contains two different kinds of terms, we may expect that, if one country doesn't follow up with another country due to a lack of industrial innovation, it can compensate producing more money or/and increase the speed of money within its financial system.

However, there are constraints in the application of these principles, since inflation or deflation can happen. It was pointed in [11] that it can correlate the spread in prices of an asset with the spread in the velocity of money with an analog of Heisenberg uncertainty relation:

$$
\sigma_{\text {price }} \times \sigma_{v m} \approx \frac{1}{\Delta t_{\text {min }}}
$$

where $\sigma_{\text {price }} \equiv\left(X_{i}-\bar{X}\right), X_{i}\left(t_{n}\right)$ is the price of an asset at a given moment of time $t_{n}=\Delta t_{\text {min }} n, n=1,2, \ldots M$ within a set of $\mathrm{M}$ time series, and $x$ is the average value of the same asset; same nomenclature applies to the spread in the velocity of money, $\sigma_{v m}$. We may interpret Eq. (13) as meaning: if the price from an average value of an asset diverges from average value during the interval of time $\Delta t_{\min }$, and the velocity of money diverges, hence the government needs to make changes in a period as long as $\Delta t_{\min }$ always looking to satisfy the uncertainty given above in Eq. (13). 
Industrial Production in the EU-core [1990=100]

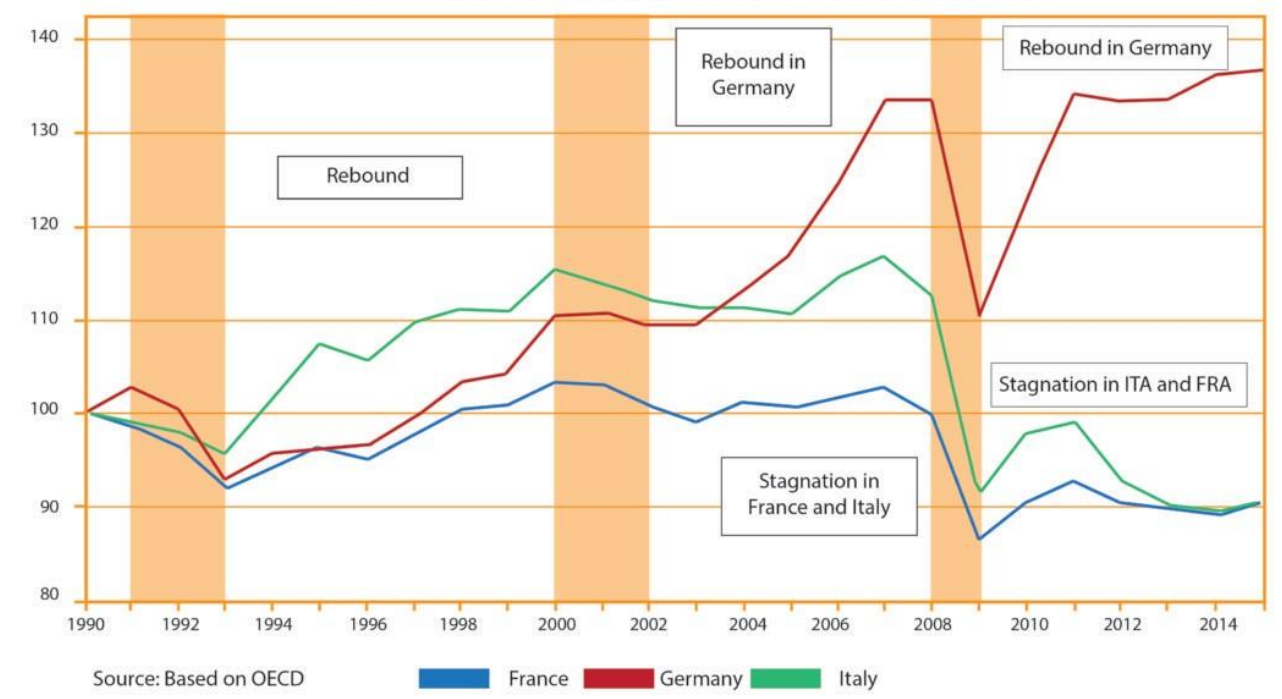

Fig. 2. Industrial production in Europe from 1990 to 2015. Courtesy: OECD.

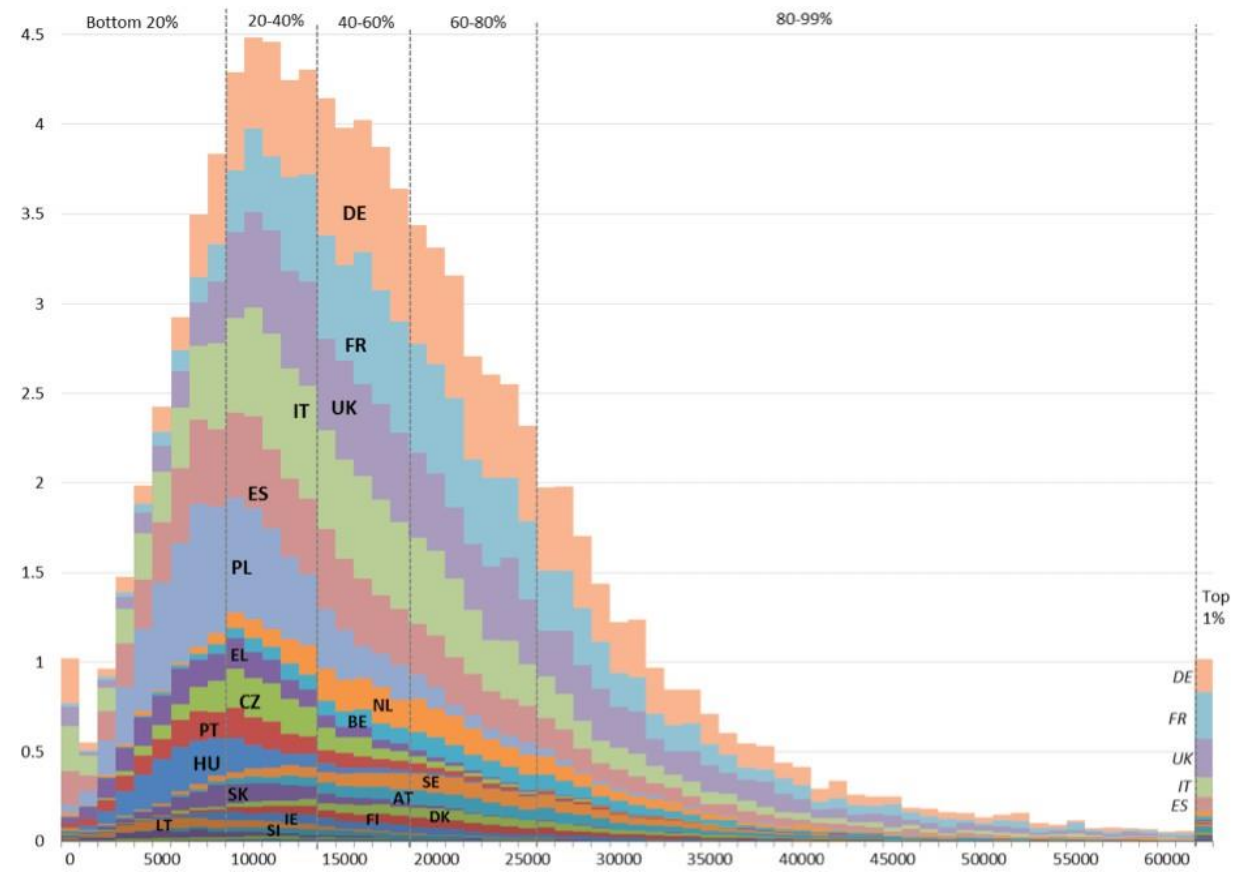

Fig. 3. EU household disposable income distribution in PPS-euro, 2013. Source: EU-SILC.

TABLE I: MEANING OF ECONOPHYSICAL QUANTITIES.

\begin{tabular}{ccc}
\hline Mechanics & Definition & Econophysics \\
\hline M-mass & money supply & $\mathrm{M}$ \\
V-velocity & velocity of money & $V_{T}$ \\
- & general price level & $\mathrm{P}$ \\
- & transactions, level of technology & $\mathrm{T}$ \\
- & real GDP & $\mathrm{Y}$ \\
$\mathrm{L}$ & angular momentum & $\mathrm{V}(\mathrm{PT})$ \\
$\mathrm{I}$ & Moment of inertia & $\mathrm{PV}$ \\
$d V / d t$ & Volume rate of change & \\
$T \Delta S$ & product of technology & $\mathrm{W}$ \\
\hline
\end{tabular}


Global income distribution in 1800,1975 , and $2010 \begin{gathered}\text { OurWorld } \\ \text { inData }\end{gathered}$

Income is measured by adjusting for price changes over time and for price differences
between countries (purchasing power parity (PPP) adjustment).
These estimates are based on reconstructed National Accounts and within-country inequality measures.

Non-market income (e.g. through home production such as subsistence farming) is taken into account.

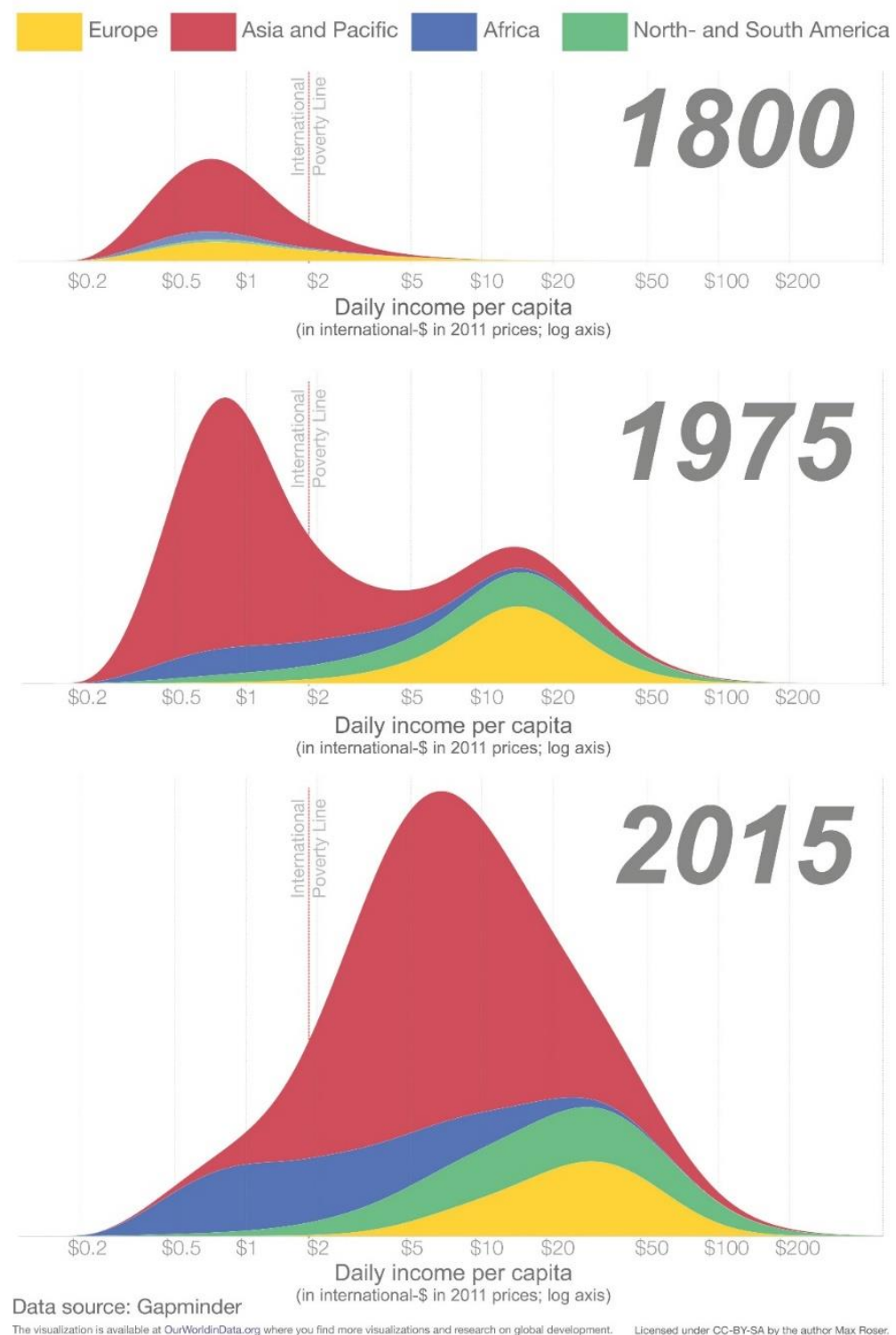

Fig. 4. Global income distribution in 1800, 1975, and 2010. Data source: Ola Rosling and published at the website of Gapminder.

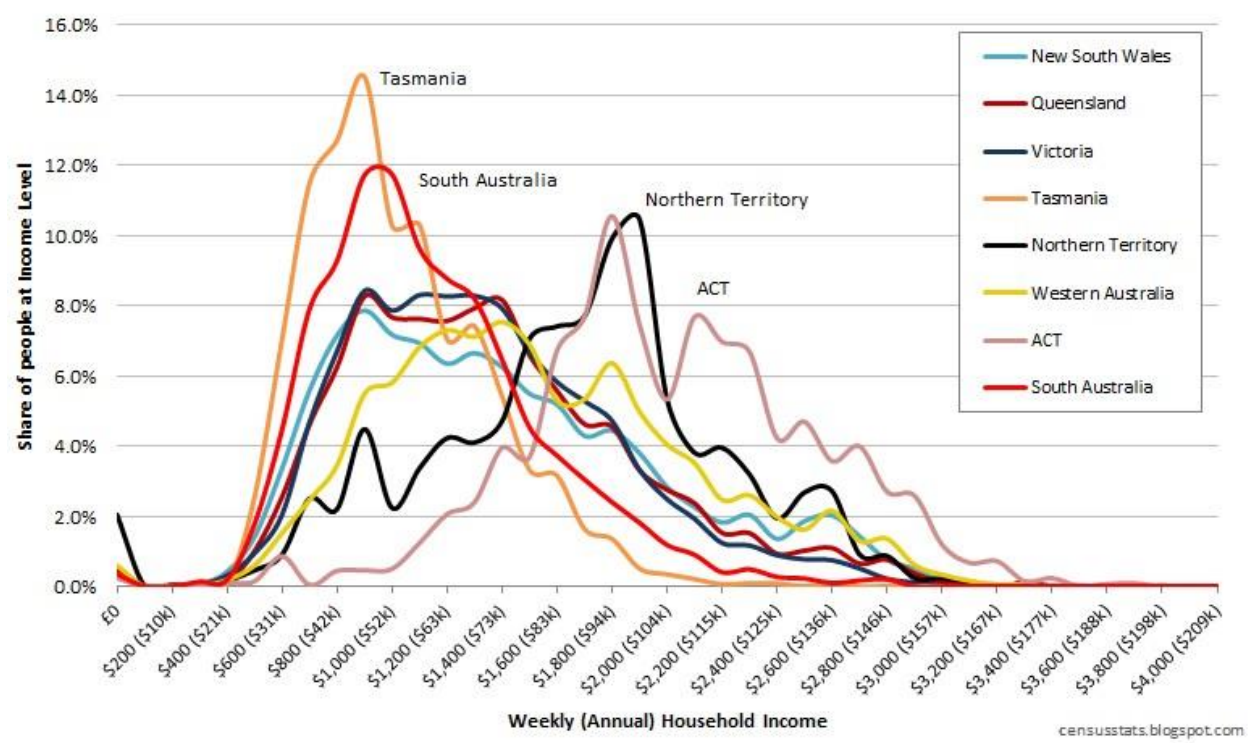

Fig. 5. Income distribution in Australian states. Source: Australia census stats. 


\section{ON WAVES OF ECONOMIC PROGRESS}

The analogy with the Boltzmann distribution function can be transposed to the field of econophysics.

For example, Fig. 3 shows the percentage of European individuals aged 15-64 (vertical axis, population) reporting different levels of equivalised ${ }^{2}$ household disposable income (horizontal axis, analog of population energy). For instance, around $4.5 \%$ of Europeans of working age have an (equivalised) household disposable income between $\mathrm{C} 10,000$ and $\mathrm{C} 11,000$ per year.

Fig. 4 exhibits the "collective" effect of international interchange in giving a kick for developing Europe, Africa, and North and South America by the extraordinary development of Asia and the Pacific. It points typically to an "energy" transfer from a mechanism that is reminiscent of the Landau-collisional effect. In the mathematical space (population distribution by daily income per capita) a wave is formed due to economic interchanges, and a group of countries joins the group velocity of the wave (in 1975), leaving behind the group velocity of Asia-Pacific and notoriously an appreciable number of the African population (due possibly to corruption and lack of interaction dynamics).

Hence, it is clear from the pictured situation (in Fig. 4) that the Asia and Pacific, Europe, Africa, and North- and South America have benefited from a progressive wave of economic development (1975), pursued until at least 2015 as shown, but less agile continents such as Africa are apparently losing momentum and possibly to a certain extent, even North- and South America. At any rate, such data denotes the existence of a coupling between the general price level and the product of technology/ velocity of money. It was shown that randomness enhances cooperation through a kind of resonance phenomenon in economic game dynamics [12], [13]. Fig. 3 shows acutely a cooperative resonant behavior where all the interacting systems have profited. However, we should note the compression with a small spread in the income in the year 1975 and the decay into two individual waves of progress for the global income distribution in the Asia and Pacific region, typical feature of a "modulation instability". This instability is the result of the political crisis in India about 1974 with a combination of political and economic crisis [14] and the period of the disastrous Cultural Revolution in China only in 1979 finished with Deng Xiaoping goal of "Four Modernizations" and further proposed the idea of "xiaokang", or "moderately prosperous society" [15].

\section{CONCLUSION}

This work aims to further elaborate the concepts of energy and entropy in the realm of econophysics to improve our understanding of economic and financial processes. It is described three types of economical instances:

1. an external driving force (e.g., regulations, supplier and sourcing instability, competitor moves, technology, customer preferences);

2. "force" that is connected to triangular arbitrage (a trading strategy that exploits the arbitrage opportunities that exist among three currencies in foreign currency exchange);

3. a "force" that is pushed by the Economic Complexity Index (or level of technology), i.e., an integrated measure of the productive aptitudes of extensive economic systems, normally cities, regions, or countries.

The practicality of the concept of momentum is also discussed in the context of an "atomistic view" of interacting countries or the ability of progressive waves to induce and transfer progress among countries. Additionally, a new technical indicator to be used in analysis of financial markets is proposed, representing cyclic rounds of the market, with it lowers and ups, the so-called "sector rotation" now widely engaged in the theory of the stock market activity.

\section{COMPETING FINANCIAL INTERESTS}

This research received no specific grant from any funding agency in the public, commercial, or not-forprofit sectors.

This work was done by the informal research team at EconoDynamics Group.

\section{CREDIT AUTHOR STATEMENT}

M. J. Pinheiro: Conceptualization, Methodology, Writing. M. R. A. Pinheiro: Software, Validation, Reviewing and Editing.

\footnotetext{
${ }^{2}$ Equivalised household income is total household income adjusted by the application of an equivalence scale to facilitate comparison of income levels between households of differing size and composition, reflecting the requirement of a larger household to have a higher level of income to achieve the same standard of living as a smaller household. Source: Australian Bureau of Statistics.
} 


\section{REFERENCES}

[1] Mario J. Pinheiro. A reformulation of mechanics and electrodynamics, Heliyon, 3 (2017) e00365.

[2] Mario J. Pinheiro. A Variational Method in Out-of-Equilibrium Physical Systems, Scientific Reports, $2013 ; 33454$.

[3] Mario J. Pinheiro. Ergontropic Dynamics: Contribution for an Extended Particle Dynamics, to appear in Rhythmic Advantages in Big Data and Machine Learning, Editors Anirban Bandyopadhyay, Kanad Ray (Springer Nature, Singapore, 2022).

[4] Matthew Yen, Daming Zhang. The Quest of Economic Temperature. Journal of Business and Economics, November 2018, 9(11):915-926.

[5] Deng et al. Crooked volatility smiles: Evidence from leveraged and inverse ETF options, Journal of Derivatives \& Hedge Funds, 2013; 19(4), 278-294.

[6] R. Merton. Option Pricing When Underlying Stock Distributions are Discontinuous. Journal of Financial Eco- nomics, 1976; 3: 771-818.

[7] César A. Hidalgo and Ricardo Hausmann. The building blocks of economic complexity. PNAS, June 30, 2009 106 (26) 1057010575.

[8] Talita Yamashiro Fordelone. Triangular Co-operation and Aid Effectiveness. Policy Dialogue on Development Co-operation (Mexico City, 28-29 September 2009).

[9] https://www.bloomberg.com/opinion/articles/2021-03-09/the-stocks-rotation-ride-is-real.

[10] https://www.macroption.com/rsi-calculation/.

[11] Soloviev V., Saptsin V. Heisenberg uncertainy principle and economic analogues of basic physical quantities. arXiv:1111.5289v1 [physics.gen-ph] 10 Nov 2011.

[12] Jie Ren, Wen-Xu Wang, and Feng Qi Randomness enhances cooperation: A resonance-type phenomenon in evolutionary games, Phys. Rev. E, 2007; 75, 045101(R).

[13] F. Gori, D. Ludovisi, P. F. Cerritelli, Forecast of oil price and consumption in the short term under three scenarios: Parabolic, linear and chaotic behaviour, Energy, 2007; 32:1291-1296.

[14] Ram Joshi. India 1974: Growing Political Crisis. Asian, Feb., 197515(2): 85-95.

[15] John Wong. Xiao-kang: Deng Xiaoping's socio-economic development target for China. Journal of Contemporary China, 1998; 7(17): 141-152.

\section{ADDITIONAL INFORMATION}

PACS: 01.75. $+m, 45.05 .+x, 45.20 . D^{-}$, 89.65.Gh

\section{APPENDIX}

APPENDIX A - Custom Java source code for the momentum indicator used with Dukascopy API

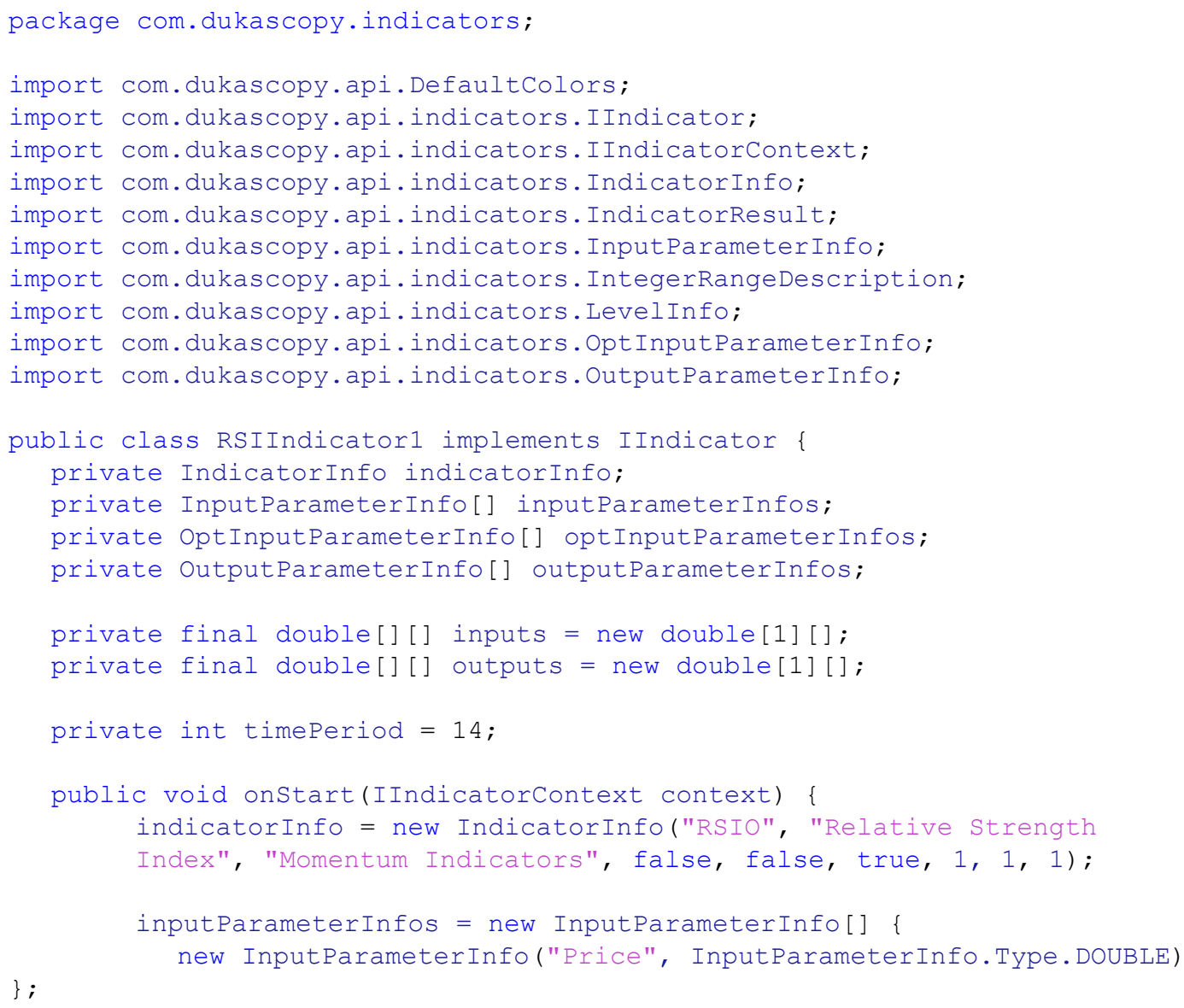


new OptInputParameterInfo("Time period",

OptInputParameterInfo.Type.OTHER, new IntegerRangeDescription(14, 2, 2000, 1))

\}

outputParameterInfos = new OutputParameterInfo[] \{

new OutputParameterInfo("out", OutputParameterInfo.Type.DOUBLE, OutputParameterInfo.Drawingstyle. LINE)

\}

indicatorInfo.setDefaultLevelsInfo(new Levelinfo[] \{

new LevelInfo("", 30, OutputParameterInfo.Drawingstyle.DASH_LINE, DefaultColors. GOLD, 1, 1)

new LevelInfo(" ", 70, OutputParameterInfo.Drawingstyle.DASH_LINE, DefaultColors.GOLD, 1, 1)

\}) ;

\}

public IndicatorResult calculate(int startIndex, int endIndex) \{

if (startIndex - getLookback ()$<0)\{$

startIndex $=$ getLookback ()

\}

if (startIndex $>$ endIndex) \{

return new IndicatorResult $(0,0)$;

\}

int $i$, today $=$ startIndex - getLookback(), outIdx $=0$;

double prevValue = inputs[0] [today], prevGain =0, prevLoss = 0, tempValue1, tempValue2;

today++;

for ( $i=$ timePeriod; $i>0$; i--)

tempValue1 = inputs[0][today++];

tempValue2 = tempValue1 - prevValue;

prevValue = tempValue1;

if ( tempValue2 $<0$ ) prevLoss $-=$ tempValue2;

else prevGain $+=$ tempValue2;

prevGain /= timePeriod;

prevLoss / = timePeriod;

if(today > startIndex) \{

tempValue1 = prevGain + prevLoss;

outputs [0] [outIdx++] = tempValue1 == 0 ? 0 : 100 * (prevGain/

tempValue1) +200* (prevGain-prevLoss) *timePeriod;

while (today $<=$ endIndex) \{

tempValue1 = inputs[0][today++];

tempValue2 = tempValue1 - prevValue;

prevValue = tempValue1;

prevLoss $\wedge=($ timePeriod -1$) ;$

prevGain $\wedge=($ timePeriod -1$) ;$

if ( tempValue2 < 0 ) prevLoss -= tempValue2;

else prevGain $+=$ tempValue2;

prevLoss /= timePeriod;

prevGain /= timePeriod;

tempValue1 = prevLoss + prevGain;

outputs [0] [outIdx++] = tempValue1 == 0 ? 0 : 100 * (prevGain /

tempValue1) +200* (prevGain-prevLoss) *timePeriod;

return new IndicatorResult (startIndex, outIdx); 


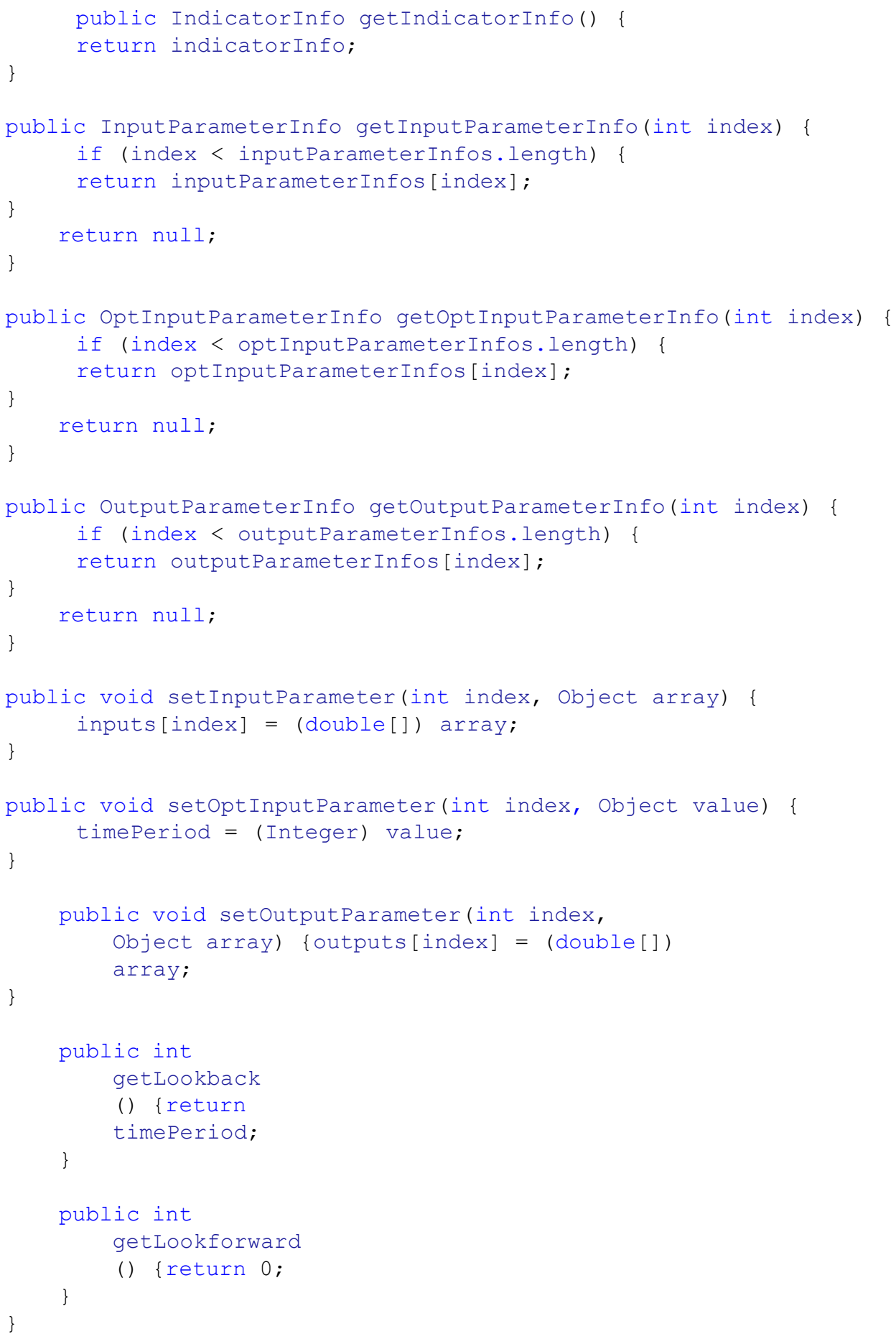

